\title{
Post-Obstructive Pneumonia in Patients with Cancer: A Review
}

\author{
Kenneth V. I. Rolston · Lior Nesher
}

Received: December 18, 2017 / Published online: February 1, 2018

(C) The Author(s) 2018. This article is an open access publication

\begin{abstract}
Published literature on post-obstructive pneumonia is difficult to find and consists mainly of case reports or small case series. This entity is encountered most often in patients with advanced lung malignancy but is also occasionally seen in patients with community-acquired pneumonia (CAP). There are substantial differences in the manifestations, treatment, and outcomes of post-obstructive pneumonia in these two settings. When obstruction is present in patients with CAP, it is almost always secondary to an underlying pulmonary malignancy. In fact, the observation of an obstructive component in patients with CAP leads to the detection of primary or metastatic lung cancer in more than $50 \%$ of such individuals. Postobstructive pneumonia in patients with
\end{abstract}

Enhanced content To view enhanced content for this article go to http://www.medengine.com/Redeem/ D66299604888602E.

K. V. I. Rolston ( $\square)$

The Department of Infectious Diseases, Infection Control, and Employee Health, MD Anderson Cancer Center, The University of Texas, Houston, TX, USA

e-mail: krolston@mdanderson.org

L. Nesher

Faculty of Health Sciences, Soroka University

Medical Center, The Infectious Disease Institute,

Ben-Gurion University of the Negev, Beersheba, Israel advanced lung malignancy is far more common ( $\sim 50 \%$ of patients) and is associated with substantial morbidity and mortality. The management of these patients is very challenging and involves multiple disciplines including medical oncology, pulmonary medicine, infectious diseases, intervention radiology, surgery, and intensive care teams. The administration of broad-spectrum antibiotic regimens is generally required. Refractory or recurrent infections despite the administration of appropriate antimicrobial therapy are the norm. Frequent and prolonged antibiotic administration leads to the development of resistant microflora. Complications such as lung abscess, empyema, and local fistula formation develop often. Relief of obstruction generally produces only temporary symptomatic improvement.

Keywords: Advanced lung cancer; Broadspectrum antimicrobial therapy; Complications; Multi-disciplinary management; Post-obstructive pneumonia; Relief of bronchial obstruction

\section{INTRODUCTION}

Pneumonia is among the leading causes of death worldwide. In the US between 5 and 10 million people develop pneumonia annually, approximately 1 million are hospitalized with 
pneumonia, and about 70,000 die every year from this disease. The association between pneumonia and pulmonary malignancies has long been recognized [1]. Several studies have documented an increased incidence of lung cancer in patients hospitalized for pneumonia compared to the population at large $[2,3]$. Studies have also demonstrated that patients 65 years of age or older and those with recurrent bouts of pneumonia are more likely to be diagnosed with pulmonary malignancy after hospitalization for pneumonia, and many clinicians recommend that such patients be closely monitored for resolution of pulmonary symptoms/ infiltrates and receive follow-up chest imaging such as computerized tomography (CT) to ensure that a pulmonary malignancy has not been missed [3-5]. Tumors of the lung and bronchus are among the most common neoplasms in man. The American Cancer Society estimates that approximately 222,500 new cases of these tumors will be diagnosed in the US in 2017 and that these tumors will be fatal in an estimated 155,870 cases in the same time period [6]. Other causes of intrinsic or extrinsic airways obstruction include malignant lymphomas, tumors of the thyroid or larynx, esophageal tumors, and metastases from extrathoracic tumors. Infections occur frequently in this patient cohort, with pneumonia (including post-obstructive pneumonia) being the leading site of infection. Post-obstructive pneumonia can occur early in the course of the pulmonary neoplasm and may occasionally be the initial manifestation that leads to its diagnosis [7]. Most cases of post-obstructive pneumonia, however, occur in patients with advanced and progressive neoplasms and are associated with considerable morbidity and mortality [8]. This review will focus on the subset of patients with community-acquired pneumonia who have features of post-obstructive pneumonia (PO$\mathrm{CAP}$ ) and compare/contrast them to patients with advanced pulmonary malignancies who develop post-obstructive pneumonia, as these are two quite distinct and separate entities.

This review article is based on previously conducted studies and does not involve any new studies of human or animal subjects performed by any of the authors.

\section{DEFINITION}

Post-obstructive pneumonia is defined as infection of the lung parenchyma distal to a bronchial obstruction [9]. There are three types of malignant airways obstruction including (1) extrinsic compression caused by an extraluminal tumor, (2) endo-bronchial obstruction caused by intraluminal tumor growth, and (3) a mixed pattern of extrinsic and endo-bronchial obatruction. This was first reported by McDonald and colleagues who described the sequence of events and changes that take place in the lung after persistent bronchial obstruction [10]. In adults such obstruction is generally due to a malignancy (most often primary but occasionally metastatic). Although this entity has been known for several years, there is very little published literature on its natural history, diagnosis, optimal treatment, and disease resolution/prognosis. It occurs in approximately $2 \%$ of people hospitalized for CAP and is usually suspected in patients with slow resolution of clinical and radiographic manifestations of CAP. However, it occurs far more frequently (40-55\%) in individuals with established pulmonary neoplasms who develop pneumonia, particularly those with advanced and progressive tumors [8].

\section{POST-OBSTRUCTIVE COMMUNITY- ACQUIRED PNEUMONIA}

As mentioned previously, PO-CAP is an uncommon condition and occurs in $\sim 2 \%$ of individuals diagnosed with $\mathrm{CAP}$, although the frequency is higher in the elderly. While infection is generally considered to be present in patients with PO-CAP, opinions vary regarding this issue, and some authors maintain that infection is infrequent in this setting $[10,11]$. To determine the frequency of bacterial infection in patients with PO-CAP and to characterize the common clinical manifestations, laboratory findings, treatment, and outcomes of PO-CAP, Abers and colleagues recently conducted a prospective study comparing patients with PO-CAP to those with proven or presumptive bacterial pneumonia (B-CAP) without 
an obstructive component over a 2-year study period [7]. The majority of patients with POCAP (93\%) were smokers and $43 \%$ had chronic obstructive pulmonary disease. The most common symptoms were fever and chills, weight loss, cough, sputum production, dyspnea, hemoptysis, and pleuritic chest pain. None of these patients had symptoms of upper respiratory infection. Leukocytosis was documented in $40 \%$ and cavitation on chest imaging was present in $17 \%$ of these patients. Microbiologic techniques documented bacterial infection in only $10 \%$ of these patients. Of note was the finding that the obstruction was secondary to a lung malignancy in all the cases of PO-CAP, and in $47 \%$ of these patients, malignancy was first discovered at the time of presentation with pneumonia.

This study revealed several differences between the two patient cohorts. Patients with PO-CAP reported a longer duration of symptoms (median duration 14 days) before admission than patients with B-CAP (median duration 5 days). Greater than 5\% weight loss was also far more frequent in patients with POCAP (68\%) than in patients with B-CAP (33\%). Additionally, patients with PO-CAP reported hemoptysis more frequently but sputum production and leukocytosis less frequently. Patients with B-CAP had lower platelet counts and higher serum procalcitonin levels. Significantly, defervescence by day 5 was more common in the B-CAP group (94\%) than in the POCAP group (60\%), and 30-day mortality was higher in the PO-CAP group (40\%) than in the B-CAP group (12\%). These findings provide strong evidence that PO-CAP is distinct from B-CAP in many ways. Recommendations partly based on the findings of this study are that patients with pneumonia who are elderly, have greater chronicity of disease, greater weight loss, hemoptysis, cavitary chest lesions, delayed response, or recurrent episodes of pneumonia might be harboring a pulmonary malignancy and need a different diagnostic approach including CT imaging and bronchoscopy if necessary.

\section{POST-OBSTRUCTIVE PNEUMONIA IN PATIENTS WITH ADVANCED PULMONARY MALIGNANCY}

There are substantial differences between POCAP and post-obstructive pneumonia that is encountered in patients with established/advanced pulmonary malignancy (PO-AM). Experience from the Infectious Diseases in-patient consultative services at our institution (an NCIdesignated Comprehensive Cancer Center) indicates that approximately $45-55 \%$ of patients with established or advanced pulmonary neoplasms who develop pneumonia have a post-obstructive component, a frequency that is much higher than that reported in CAP [8]. This frequency may actually be an underestimate as we are not consulted on every patient with advanced lung cancer and pneumonia. Nevertheless, it does suggest that PO$\mathrm{AM}$ is relatively common in this setting. The initial event in the development of PO-AM is the retention of mucus distal to the obstruction followed by filling of the alveoli with mucus and serum exuded from alveolar capillaries. As the obstruction persists or advances, infection accompanied by an acute inflammatory (neutrophilic) response develops. The vast majority of patients with PO-AM at this stage $(>85 \%)$ are febrile and have a productive, generally purulent cough (unless the involved airway is almost completely obstructed by the tumor in which case the cough is non-productive). Other common manifestations include dyspnea, pleuritic chest pain, hemoptysis, significant weight loss, loss of appetite, and cachexia (Table 1). Most patients have moderate leukocytosis (unless they have chemotherapy-related neutropenia). Microbiologic specimens are often difficult to obtain since the infection is located distal to the obstruction. As mentioned previously, a substantial proportion of patients may not produce sputum. Additionally, sputum cultures often represent bacterial colonization of distal airways and not necessarily the pathogen(s) responsible for the infection $[12,13]$. Some authors have documented a discordance between sputum cultures and cultures obtained by ultrasoundguided trans-thoracic needle aspiration of tissue 
Table 1 Common clinical features in patients with advanced lung malignancies and post-obstructive pneumonia

\begin{tabular}{lc}
\hline Clinical features & \% Frequency \\
\hline Fever & $80-85$ \\
Dyspnea & $>90$ \\
Cough & $>90$ \\
Hemoptysis & $10-30$ \\
Chest pain & $10-40$ \\
Weight loss & $>70$ \\
Loss of appetite & $>70$ \\
Cachexia & $>50$ \\
\hline
\end{tabular}

Data are from the infectious diseases consultative services at the University of Texas, MD Anderson Cancer Center, Houston, Texas, USA

distal to the obstruction $[14,15]$. When reliable samples are available (needle aspiration or BAL), the microbiology generally reveals polymicrobial flora $[8,16]$. Organisms isolated most frequently are Staphylococcus species (including MRSA), Streptococcus species [including viridans group streptococci (VGS) and beta-hemolytic streptococci], the Enterobacteriaceae, Pseudomonas aeruginosa, and various anaerobes (Table 2). Candida species are also recovered frequently, but their clinical significance is unclear, and it is commonly believed that they most often represent oro-pharyngeal colonization.

Antimicrobial therapy of PO-AM consists of the administration of broad-spectrum antimicrobial regimens that provide coverage against the anticipated pathogens listed above and should be based on local/institutional microbiologic data and susceptibility/resistance patterns. Due to the presence of obstruction, response to antimicrobial therapy is often slow and incomplete, and recurrent infections are frequent, leading to prolonged and repeated use of antimicrobial agents. This in turn leads to the development or selection of organisms resistant to commonly used antimicrobial agents. At our institution, approximately $70 \%$ of S. aureus isolates are methicillin-resistant, and approximately $40-60 \%$ of VGS isolates are penicillin non-susceptible [17, 18]. Extended-spectrum beta-lactamase (ESBL)-producing gram-negative bacteria such as E. coli are also relatively common in this setting. Carbapenem-resistant Enterobacteriaceae (CRE) are less common but may be a problem at specific institutions [19]. Occasionally multidrug-resistant organisms such as $P$. aeruginosa and Stenotrophomonas maltophilia and Acinetobacter spp. are isolated $[20,21]$. Due to the relative frequency of MRSAand ESBL-producing organisms at our institution, initial combination therapy with an agent such as vancomycin or linezolid (for MRSA coverage) and a carbapenem such as imipenem/cilastatin or meropenem (for coverage against ESBL producers and anaerobes) is generally used. In patients with positive microbiology/susceptibility data, the initial regimen can be modified if necessary. Recommendations for antimicrobial therapy of PO-AM are listed in Table 3. Some clinicians occasionally utilize aerosolized antibiotics (most commonly the aminoglycosides or fluoroquinolones) in addition to systemic agents in this setting although the efficacy of this approach has not been fully demonstrated. The optimal duration of treatment for PO-AM has also not been established, and, as previously mentioned, prolonged and/ or repeated courses of antimicrobial therapy are the norm. Thus, a vicious cycle of prolonged, broad-spectrum antibiotic therapy leading to the development of resistance is perpetuated.

In patients with incomplete responses or recurrent infections, relief of the obstruction is necessary. Several options to try and achieve this are currently available. These include various endobronchial treatment options such as brachytherapy, laser therapy, electrocautery, cryotherapy, argon plasma coagulation, and photodynamic therapy, with or without airway stents [22, 23]. No single modality is ideal, and often the modality chosen depends on the site of the obstruction and local expertise or preferences.

Endobronchial brachytherapy is often used for palliation of symptoms associated with endobronchial obstruction. Irradiation can be delivered with low-dose-rate brachytherapy 
Table 2 Microbiologic findings in cancer patients with post-obstructive pneumonia

Gram-positive organisms

Staphylococcus aureus (including MRSA)

Viridans group streptococci ( 60\% penicillin non-susceptible)

Beta-hemolytic streptococci (groups A, B, C, F, and G)

Gram-negative organisms

Escherichia coli

Klebsiella species ${ }^{\mathrm{a}}$

Other Enterobacteriaceae ${ }^{\mathrm{a}}$

Pseudomonas aeruginosa ${ }^{\mathrm{b}}$

Strenotrophomonas maltophilia ${ }^{\mathrm{b}}$

Acinetobacter species ${ }^{\mathrm{b}}$

Other NFGNB ${ }^{\mathrm{c}}$

Anaerobes

Peptococcus spp. and Peptostreptococcus spp.

Fusobacterium nucleatum

Bacteroides melaninogenicus

Fungi (Candida species)

Most studies report predominantly polymicrobial flora

a Including extended-spectrum beta-lactamase (ESBL) producers and carbapenem resistant Enterobacteriaceae

b These organisms are often multidrug resistant

c NFGNB: non-fermentative gram-negative bacilli

(i.e., one treatment delivered over hours or days) or as high-dose-rate brachytherapy (fractionated treatment delivered in a few minutes). A recent study evaluating high-dose-rate brachytherapy reported that bronchial obstruction was improved in $73.4 \%$ of patients, and $80 \%$ of patients with PO-AM responded with relief of symptoms [24]. There was good patient tolerance, excellent patient compliance, a low complication rate, and substantial improvement in the quality of life. This is an acceptable option in most institutions.

Laser resection using flexible or rigid bronchoscopes is another method of relieving bronchial obstruction. There are several different types of lasers, but the one used most commonly is the neodymium:yttrium aluminum garnet (Nd-YAG) equipment [22]. Another type of laser, the holmium:YAG laser, has also been used in this setting. Squires and colleagues recently reported symptomatic improvement in $77 \%$ of patients with malignant obstruction who received holmium:YAG laser therapy [25]. Complications occurred in only $2.3 \%$ of the procedures, and mortality was seen in less than $1 \%$ of procedures. Laser bronchoscopy is used most often for obstructive lesions of the trachea, the right and left main bronchi, and the bronchus intermedius. In many cases, laser bronchoscopy is used in combination with other modalities such as brachytherapy, stenting, and external beam irradiation. 
Table 3 Recommended antibiotics for the treatment of post-obstructive pneumonia

Broad-spectrum agents (may be used as monotherapy)

Piperacillin/tazobactam

Carbapenem (imipenem/meropenem/doripenem)

Narrow-spectrum agents (need to be used in combination)

Respiratory quinolones

Cefepime

Ceftazidime

Ertapenem

Vancomycin

Linezolid

Tigecycline

Amoxicillin/clavulanate

Ampicillin/sulbactam

Clindamycin

Colistin $^{\mathrm{a}}$

Trimethoprim/sulfamethoxazole ${ }^{\mathrm{b}}$

Newer agents

Ceftazidime/avibactam

Ceftolozane/tazobactam

Meropenem/vaborbactam

Imipenem-cilastatin/relebactam ${ }^{c}$

Aztreonam/avibactam ${ }^{c}$

Cefiderocol $^{\mathrm{c}}$

${ }^{a}$ Consider adding colistin for resistant pathogens such as Acinetobacter spp. in institutions with high prevalence

b Consider adding coverage for Stenotrophomonas maltophilia in patients with prior exposure to carbapenems

${ }^{c}$ Have not been approved yet for clinical use but are in advanced stages of development

Argon plasma coagulation (APC) is a type of noncontact electrocoagulation [26]. APC delivers high-frequency current via a flexible probe, utilizing electrically conductive argon plasma as a medium of delivery. This has now become one of the most commonly used modalities for the treatment or palliation of airways obstruction $[27,28]$. It produces immediate airways patency and relief of symptoms in the majority of patients $(<90 \%)$, is generally easy and safe to perform at the bedside or even in outpatient settings, and is well tolerated by most patients even after repeated applications. The frequency of procedure-related complications is low including the frequency of post-procedure bacteremia [29].

Stents are in use primarily for counteracting extrinsic compression of airways or maintaining airway patency after endoscopic removal of intraluminal tumors. Stents are also used in 
patients who develop fistulas (e.g., trachea-esophageal fistula) [22]. Many polymer stents (e.g., silicone stents) and stents made of various metals such as steel are available. Drug-eluting stents akin to those in use for coronary care are also in use [30]. There appears to be no clear advantage of any type of stent over another although no randomized studies comparing the various types of stents have been conducted. The location, length, and shape of the stenosis are important considerations in determining the type of stent used.

One recent large retrospective observational study described the use of various therapeutic interventions for malignant airway obstruction [31]. Over 7 years, 802 rigid bronchoscopic procedures were performed in 547 patients with malignant airway obstruction. Argon plasma coagulation was used in 373 procedures (257 patients), total laser application was performed in 250 procedures (178 patients), stents were applied during 171 procedures (147 patients), and cryotherapy was used in 93 procedures (54 patients). This study showed that all patients had substantial and rapid relief of symptoms following endo-bronchial treatment and stenting. There was improved quality of life and some additional time for the administration of adjuvant chemoradiation. Complications secondary to stent placement included re-obstruction of the lumen by tumor, stent migration, mucus plugging, and occasional airways perforation, especially when used in combination with laser. Argon plasma coagulation was used more often for lesions involving both the trachea and a main bronchus. This modality is a non-contact mode of treatment and provided immediate symptomatic relief with few complications. Cryotherapy was used less often and has a delayed mode of action compared to other modalities. There was no consensus on the factors influencing the choice of interventional procedure or modality used.

Another large multicenter study evaluated the success of therapeutic bronchoscopy for malignant central airways obstruction [32]. This study was conducted at 15 centers and included 1115 procedures in 947 patients. Most procedures (93\%) were technically successful with success being defined as $>50 \%$ reopening of the airway lumen. The individual center success rate ranged from 90 to $98 \%$, indicating relative uniformity. On multivariate analysis, endobronchial obstruction and stent placement were associated with higher technical success rates, whereas American Society of Anesthesiology (ASA) score $>3$, renal failure, primary lung cancer, left main stem disease, and tracheaesophageal fistulae were associated with lower success rates. Patients with the most dyspnea and the lowest functional status benefited the most. Consequently, one of the conclusions of this study was that patients with severe functional impairment should not be denied therapeutic bronchoscopy based on perceived risk. This study also failed to identify any single ablative modality and/or type of stent being superior to any other.

Serious complications such as lung abscess, empyema, hemorrhage, and fistula formation (broncho-esophageal or trachea-esophageal) occur in $\sim 10$ to $15 \%$ of patients with PO-AM [8]. These can result in considerable morbidity and in delays in the administration of antineoplastic therapy that can have a negative impact on overall outcome. Several recent reports have documented an increased frequency of tracheaesophageal fistula formation in patients treated with chemoradiation and bevacizumab [33-35]. Most of these complications require some sort of surgical intervention in addition to specific medical management and supportive care. Despite these measures, the outcome is often unsatisfactory [36-38].

\section{ILLUSTRATIVE CLINICAL CASE}

A 63-year-old female suddenly developed severe dyspnea and presented to our Emergency Department (ED) $24 \mathrm{~h}$ later. She also had a dry cough but had no chest pain or hemoptysis. In the ED she was documented to be febrile with a temperature of $38.8^{\circ} \mathrm{C}$. Physical examination revealed absent breath sounds in the right upper and middle lobes. Laboratory data showed the presence of leukocytosis (WBC count $17,400 / \mathrm{mm}^{3}$ ). Imaging of the chest (CT) revealed a right hilar mass with mediastinal invasion and complete obstruction and collapse 
of the right upper lobe and near complete obstruction of the right main stem bronchus. Post-obstructive pneumonia was considered very likely and she was placed on empiric, broad-spectrum, parenteral antibiotics (vancomycin and cefepime). She also underwent rigid bronchoscopy with tumor debulking and stenting of the right main stem bronchus, resulting in complete patency of the right middle lobe and right lower lobe. Re-expansion of the right upper lobe was not achieved. Biopsies taken at the time of the rigid bronchoscopy revealed the presence of non-small cell lung carcinoma (NSCLC). The patient remained clinically stable for approximately 10 days. She then developed progressive dyspnea and hypoxia and needed to be transferred to the intensive care unit (ICU) for the management of respiratory insufficiency. Repeat chest imaging showed a new, large, right-sided pleural effusion. She underwent thoracentesis with removal of $750 \mathrm{ml}$ of amber-colored fluid. This only led to temporary relief of symptoms. She subsequently had an intrapleural drainage catheter placed, with an additional $1900 \mathrm{ml}$ of fluid being drained. Both fluid samples were positive for Pseudomonas aeruginosa indicating the presence of empyema. The organism was susceptible to cefepime, amikacin, meropenem, ciprofloxacin, and piperacillin/tazobactam. Therapy was changed to piperacillin/tazobactam plus ciprofloxacin to which the patient responded. This case demonstrated the complex nature of the management of patients with PO$\mathrm{AM}$ and its complications. It illustrates the need to involve multiple services including medical oncology, pulmonary medicine, infectious diseases, and the ICU team. (Table 4).

\section{SUMMARY}

A small proportion of patients with community-acquired pneumonia $(\sim 2 \%)$ have an obstructive component. This is most often due to an underlying, often undetected tumor. Although delayed responses to antimicrobial therapy and recurrent infections may occur, the infection resolves in most patients. In contrast, post-obstructive pneumonia in patients with advanced lung cancer is a serious, often lifethreatening development and occurs in approximately $50 \%$ of such patients. Most patients are treated with broad-spectrum antimicrobial regimens based on anticipated pathogens in this setting. Nevertheless, these infections seldom resolve completely, and recurrent or refractory infections are common. Other serious complications such as lung abscess, empyema, and fistula formation occur in this setting. These are primarily due to persistent or progressive obstruction. Many modalities to overcome obstruction are available. No one modality is superior to the rest,

Table 4 Key recommendations for the management of post-obstructive pneumonia in patients with advanced lung cancer

Multidisciplinary approach with early involvement of various specialties (medical oncology, pulmonary medicine, infectious diseases, intervention radiology, surgery, and intensive care teams)

Administer empiric broad-spectrum antimicrobial therapy against anticipated pathogens (staphylococci, streptococci, Enterobacteriaceae, NFGNB, ${ }^{a}$ and anaerobes)

Monitor for the emergence of resistant pathogens and modify treatment accordingly

Attempt to overcome obstruction as soon as possible (often patients with severe symptoms deemed to be at high risk derive the most benefit from such interventions)

Specific antimicrobial regimens should be based on local epidemiologic data and susceptibility/resistance patterns

These recommendations are based on a review of the current literature and personal experience at a comprehensive cancer center

${ }^{a}$ NFGNB: non-fermentative gram-negative bacilli 
and often multiple modalities are used in the same patient. Unfortunately, these measures are generally palliative, and the overall outcome is poor.

\section{ACKNOWLEDGEMENTS}

Funding. No funding or sponsorship was received for conducting or publication of this review article.

Authorship. All named authors meet the International Committee of Medical Journal Editors (ICMJE) criteria for authorship for this manuscript, take responsibility for the integrity of the work as a whole, and have given final approval for the version to be published.

Disclosures. Kenneth V.I. Rolston and Lior Nesher have nothing to disclose.

Compliance with ethics guidelines. This review article is based on previously conducted studies and does not involve any new studies of human or animal subjects performed by any of the authors.

Open Access. This article is distributed under the terms of the Creative Commons Attribution-NonCommercial 4.0 International License (http://creativecommons.org/licenses/ by-nc/4.0/), which permits any noncommercial use, distribution, and reproduction in any medium, provided you give appropriate credit to the original author(s) and the source, provide a link to the Creative Commons license, and indicate if changes were made.

\section{REFERENCES}

1. Marrie TJ. Pneumonia and carcinoma of the lung. J Infect. 1994;29:45-52.

2. Søyseth V, Benth JS, Stavem K. The association between hospitalisation for pneumonia and the diagnosis of lung cancer. Lung Cancer. 2007;57:152-8.
3. Mortensen EM, Copeland LA, Pugh MJ, et al. Diagnosis of pulmonary malignancy after hospitalization for pneumonia. Am J Med. 2010;123:66-71.

4. Cunha BA, Syed U, Mikail N. Bronchoalveolar carcinoma (adenocarcinoma) mimicking recurrent bacterial community-acquired pneumonia (CAP). Heart Lung. 2012;41:83-6.

5. Holmberg H, Kragsbjerg P. Association of pneumonia and lung cancer: the value of convalescent chest radiography and follow-up. Scand J Infect Dis. 1993;25:93-100.

6. Siegel RL, Miller KD, Jemal A. Cancer statistics, 2017. CA Cancer J Clin. 2017;67:7-30.

7. Abers MS, Sandvall BP, Sampath R, et al. Postobstructive pneumonia: an underdescribed syndrome. Clin Infect Dis. 2016;62:957-61.

8. Rolston KV. Postobstructive pneumonia in cancer patients. Clin Infect Dis. 2016;63:707-8.

9. Torres A, Ferrer M. Editorial commentary: distinguishing postobstructive lung infection from community-acquired pneumonia. Clin Infect Dis. 2016;62:962-3.

10. Mcdonald JR, Harrington SW, Clagett OT. Obstructive pneumonitis of neoplastic origin; an interpretation of one form of so-called atelectasis and its correlation according to presence of absence of sputum. J Thorac Surg. 1949;18:97-112.

11. Burke M, Fraser R. Obstructive pneumonitis: a pathologic and pathogenetic reappraisal. Radiology. 1988;166:699-704.

12. Cabello H, Torres A, Celis R, et al. Bacterial colonization of distal airways in healthy subjects and chronic lung disease: a bronchoscopic study. Eur Respir J. 1997;10:1137-44.

13. Ioanas M, Angrill J, Baldo X, et al. Bronchial bacterial colonization in patients with resectable lung carcinoma. Eur Respir J. 2002;19:326-32.

14. Liaw YS, Yang PC, Wu ZG, et al. The bacteriology of obstructive pneumonitis. A prospective study using ultrasound-guided transthoracic needle aspiration. Am J Respir Crit Care Med. 1994;149:1648-53.

15. Liao WY, Liaw YS, Wang HC, Chen KY, Luh KT, Yang PC. Bacteriology of infected cavitating lung tumor. Am J Respir Crit Care Med. 2000;161:1750-3.

16. Hsu-Kim C, Hoag JB, Cheng GS, Lund ME. The microbiology of postobstructive pneumonia in lung cancer patients. J Bronchology Interv Pulmonol. 2013;20:266-70. 
17. Rolston KV, Jamal MA, Nesher L, Shelburne SA, Raad I, Prince RA. In vitro activity of ceftaroline and comparator agents against Gram-positive and Gram-negative clinical isolates from cancer patients. Int J Antimicrob Agents. 2017;49:416-21.

18. Han XY, Kamana M, Rolston KV. Viridans streptococci isolated by culture from blood of cancer patients: clinical and microbiologic analysis of 50 cases. J Clin Microbiol. 2006;44:160-5.

19. Aitken SL, Tarrand JJ, Deshpande LM, et al. high rates of nonsusceptibility to ceftazidime-avibactam and identification of New Delhi metallo- $\beta$-lactamase production in Enterobacteriaceae bloodstream infections at a major cancer center. Clin Infect Dis. 2016;63:954-8.

20. Rolston KV, Bodey GP, Safdar A. Polymicrobial infection in patients with cancer: an underappreciated and underreported entity. Clin Infect Dis. 2007;45:228-33.

21. Rolston KV. Polymicrobial pulmonary infections in cancer patients with underlying solid tumors. Infection. 2017;45:245-6.

22. Bolliger CT, Sutedja TG, Strausz J, Freitag L. Therapeutic bronchoscopy with immediate effect: laser, electrocautery, argon plasma coagulation and stents. Eur Respir J. 2006;27:1258-71.

23. Guibert N, Mhanna L, Droneau S, et al. Techniques of endoscopic airway tumor treatment. J Thorac Dis. 2016;8:3343-60.

24. de Aquino Gorayeb MM, Gregório MG, de Oliveira EQ, Aisen S. Carvalho HeA. High-dose-rate brachytherapy in symptom palliation due to malignant endobronchial obstruction: a quantitative assessment. Brachytherapy. 2013;12:471-8.

25. Squiers JJ, Teeter WA, Hoopman JE, et al. Holmiu$\mathrm{m}$ :YAG laser bronchoscopy ablation of benign and malignant airway obstructions: an 8-year experience. Lasers Med Sci. 2014;29:1437-43.

26. Tremblay A, Marquette CH. Endobronchial electrocautery and argon plasma coagulation: a practical approach. Can Respir J. 2004;11:305-10.

27. Crosta C, Spaggiari L, De Stefano A, Fiori G, Ravizza D, Pastorino U. Endoscopic argon plasma coagulation for palliative treatment of malignant airway obstructions: early results in 47 cases. Lung Cancer. 2001;33:75-80.
28. Morice RC, Ece T, Ece F, Keus L. Endobronchial argon plasma coagulation for treatment of hemoptysis and neoplastic airway obstruction. Chest. 2001;119:781-7.

29. Matveychuk A, Guber A, Talker O, Shitrit D. Incidence of bacteremia following bronchoscopy with argon plasma coagulation: a prospective study. Lung. 2014;192:615-8.

30. Hohenforst-Schmidt W, Zarogoulidis P, Pitsiou G, et al. Drug eluting stents for malignant airway obstruction: a critical review of the literature. J Cancer. 2016;7:377-90.

31. Dalar L, Özdemir C, Abul Y, et al. Therapeutic bronchoscopic interventions for malignant airway obstruction: a retrospective study from experience on 547 patients. Medicine (Baltimore). 2016;95:e3886.

32. Ost DE, Ernst A, Grosu HB, et al. Therapeutic bronchoscopy for malignant central airway obstruction: success rates and impact on dyspnea and quality of life. Chest. 2015;147:1282-98.

33. Spigel DR, Hainsworth JD, Yardley DA, et al. Tracheoesophageal fistula formation in patients with lung cancer treated with chemoradiation and bevacizumab. J Clin Oncol. 2010;28:43-8.

34. Goodgame B, Veeramachaneni N, Patterson A, Govindan R. Tracheo-esophageal fistula with bevacizumab after mediastinal radiation. J Thorac Oncol. 2008;3:1080-1.

35. Schreiber J, Waldburg N. Bronchoesophageal fistula and fatal hemoptysis after bevacizumab-containing chemotherapy without radiation in lung cancer. J Clin Oncol. 2012;30:e324.

36. Zhou C, Hu Y, Xiao Y, Yin W. Current treatment of tracheoesophageal fistula. Ther Adv Respir Dis. 2017;11:173-80.

37. Su JW, Mason DP, Murthy SC, Rice TW. Closure of a large tracheoesophageal fistula using AlloDerm. J Thorac Cardiovasc Surg. 2008;135:706-7.

38. Kvale PA, Selecky PA, Prakash UB. Physicians ACoC. Palliative care in lung cancer: ACCP evidence-based clinical practice guidelines (2nd edition). Chest. 2007;132:368S-403S. 